\title{
Compiler Generation and Autotuning of Communication-Avoiding Operators for Geometric Multigrid
}

\author{
Protonu Basu \\ Anand Venkat \\ Mary Hall \\ University of Utah \\ Salt Lake City, Utah 84112
}

\author{
Samuel Williams \\ Brian Van Straalen \\ Leonid Oliker \\ Lawrence Berkeley National Laboratory \\ Berkeley, CA 94720
}

\begin{abstract}
This paper describes a compiler approach to communicationavoiding optimizations in geometric multigrid (GMG), one of the most popular methods for solving partial differential equations. Communication-avoiding optimizations reduce vertical communication through the memory hierarchy and horizontal communication across processes or threads, usually at the expense of introducing redundant computation. We focus on applying these optimizations to the smooth operator, which successively reduces the error and accounts for the largest fraction of the GMG execution time. Our compiler technology applies both novel and known transformations to derive an implementation comparable to manually-tuned code. To make the approach portable, an underlying autotuning system explores the tradeoff between reduced communication and increased computation, as well as tradeoffs in threading schemes, to automatically identify the best implementation for a particular architecture and at each computation phase. Results show that we are able to quadruple the performance of the smooth operation on the finest grids while attaining similar or better performance than manually-tuned code.
\end{abstract}

Categories and Subject Descriptors D.3.4 [Programming Languages]: Compilers, Optimization, Code Generation

Keywords Geometric Multigrid, Compiler Optimization, Autotuning

\section{Introduction}

Geometric multigrid (GMG) is an important family of algorithms used by computational scientists to accelerate the convergence of iterative solvers for linear systems. In GMG, floating-point computation is dwarfed by the overhead of data movement, making managing the memory hierarchy and cross-processor communication critical to achieving high performance. Prior work on optimizing the stencil computations that are contained within GMG have led to techniques like cache oblivious algorithms, time skewing, wavefront optimizations and overlapped tiling [7, 10, 11, 17, 18, 21, 25, $26,31,32]$. For many of these efforts, the problems were simplified as compared to real-world applications, using 2-dimensional or constant-coefficient stencils without control flow and starting from a sequential specification rather than parallel specification.

As modern architectures continue to grow in core count and exhibit a hierarchy of complex inter-thread and inter-process interactions, new communication-avoiding techniques have been introduced for GMG that encapsulate several of the optimizations mentioned above. Communication-avoiding optimizations reduce vertical communication through the memory hierarchy and horizon- tal communication across processes or threads, usually at the expense of introducing redundant computation. Programmers generally need to introduce these optimizations manually, while attempting to discern the optimal combination of parameters, thus resulting in a significant growth in code complexity and non-portability across different architectures. This paper describes how to perform these optimizations automatically by a compiler, generating highperforming code from a relatively straightforward expression of a set of operators in a scalable MPI implementation. Our starting point is the miniGMG benchmark that is intended to represent use of GMG in real-world scalable applications.

We focus on optimizations required for the smooth operator, the most compute-intensive portion of GMG. Vertical communicationavoiding optimizations necessitate the support for data-flow analysis, which must be incorporated into the transformations to enable (1) fusing several operators so that intermediate data remains in cache from definition to use; (2) avoiding writes back to memory of temporary data; as well as (3) creating a wavefront so that multiple planes can share data in cache with a minimal cache working set. A horizontal communication-avoiding optimization adds ghost zones to reduce the frequency of inter-processor communication at the expense of redundant computation. While most of these optimizations rely on composing standard loop transformations, most would generally not be implemented in a standard compiler as they are either domain-specific (introduction of ghost zones), or specifically effective for GMG classes of computations.

The compiler system of this research effort employs autotuning to make these optimizations portable across different architectures. Autotuning systems employ empirical techniques to evaluate the suitability of a search space of possible implementations of a computation. Communication-avoiding optimizations such as increasing ghost zone depth tradeoff increased computation for less frequent communication. The wavefront optimization exploits reuse deeper in the memory hierarchy (L1/L2), but risks exceeding capacity limitations if applied too aggressively. The granularity of profitable thread-level parallelism depends on both architecture and the level in the GMG V-cycle. Through autotuning, these tradeoffs can be explored to select the context-specific optimal solutions, across a variety of architectures.

The main contribution of this work is the first exploration of compiler-directed communication-avoiding optimization for GMG. As compared to prior research on domain-specific compilers for the stencil computations that are included within GMG [13, $22,33]$, our work more closely addresses the needs of real-world applications because it optimizes in the context of an existing scalable parallel benchmark (miniGMG), and it examines the complex 


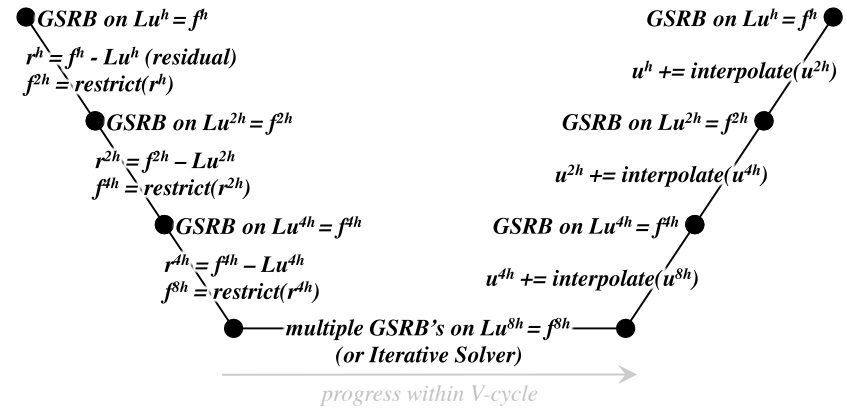

Figure 1. The Multigrid V-cycle for solving $L u^{h}=f^{h}$. Superscripts represent grid spacing. For large problems, a highperformance, iterative solver is employed at the bottom (coarsest grids).

and more representative operator Gauss-Seidel Red Black rather than the simpler Jacobi. Additionally, we enable autotuning across the ghost zone depth as well as the tuning strategies, thus allowing the automation of differing optimization schemes across individual levels of the V-cycle. This infrastructure is therefore adaptable to next-generation platforms with increasing memory-hierarchy and threading complexity. Overall, we demonstrate portability with a $4 \mathrm{x}$ improvement for the most time consuming smooth of the Vcycle, while attaining the performance of previously published, high performance hand-tuned code[29].

\section{Geometric Multigrid}

Multigrid solvers calculate a correction to the solution at the current grid resolution using a solution on a coarser grid. This process may be expressed recursively. Geometric multigrid (GMG) begins with a structured mesh, where each progressively coarser grid contains half the grid points in each dimension. Given the fact that the operators are the same irrespective of grid spacing, this exponential reduction in grid sizes can bound multigrid's computational complexity to $\mathrm{O}(\mathrm{N})$ where $\mathrm{N}$ is the number of variables. When performance is highly correlated to computational complexity, the time spent on the finer grids will dominate the run time.

Figure 1 visualizes the structure of a multigrid V-cycle for solving $L u^{h}=f^{h}$ in which $L$ is the operator, $u$ is the solution, $f$ is the right-hand side, and superscripts represent grid spacings. At each grid spacing, multiple smooth operators reduce the error in the solution. The smooth can be a simple relaxation such as Jacobi, or something more complex like a Gauss-Seidel, Red-Black (GSRB).

The right-hand side of the next coarser grid is defined as the restriction of the residual $\left(f^{h}-L u^{h}\right)$. Eventually, the grid (or collection of grids) cannot be coarsened any further using geometric multigrid. At that point, most algorithms switch to a bottom solver that can be as simple as multiple relaxations or as complicated as algebraic multigrid, a Krylov iterative solver, or a direct sparse solver. Once the coarsest grid is solved, the multigrid algorithm applies the solution (a correction) to progressively finer grids. This requires an interpolation of $u^{2 h}$ onto $u^{h}$. At each level, a smooth operator is applied to the new correction.

For problems on relatively few nodes, the performance of smooth on the finer grids dominates the run time. In this paper, we therefore focus on the smooth bottleneck, optimizing both the simpler Jacobi that is common to compiler papers [13, 16, 21], and the more complex Gauss-Seidel, Red-Black (GSRB), which predominates real-word applications and requires data-flow analysis and other support for control flow. Overall we demonstrate that our compiler infrastructure can successively optimize both of
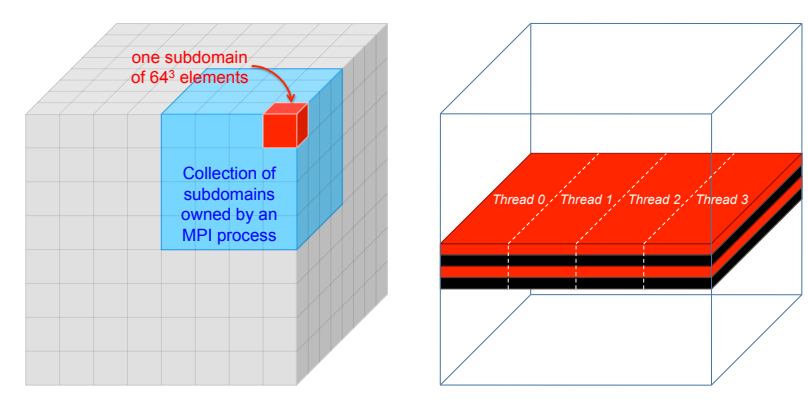

Figure 2. Visualization of the domain/process/subdomain hierarchy in miniGMG (left) and the threaded GSRB wavefront strategy within the application of $\operatorname{smooth}()$ to each subdomain (right).

these relaxation techniques and deliver high performance across our evaluated platforms.

\section{1 miniGMG Benchmark}

Our work builds on the compact multigrid solver benchmark of Williams et al. [29], called miniGMG. As shown in Figure 2(left), the miniGMG benchmark creates a global 3D domain, and partitions it into subdomains of sizes similar to those found in real-world AMR multigrid applications. The list of subdomains is then partitioned amongst MPI processes. All subdomains must exchange ghost zones after each computation phase, via an MPI call. However, when on the same node, the code is optimized to perform a buffer copy.

To provide direct comparisons to [29], we use the same doubleprecision, finite volume discretization of the variable-coefficient operator $a \vec{\alpha} I-b \nabla \vec{\beta} \nabla$, with the same periodic boundary conditions, and replicate the $256^{3}$ problem (per compute node) on all platforms. Developers often wish to maintain flexibility and thus create smooth operators by composing multiple simpler operators, as captured in the excerpt of the baseline miniGMG benchmark shown in Figure 3. In this code, the Helmholtz operator requires calculation of the Laplacian. Thus, the smooth operator in the input code calculates the Laplacian, Helmholtz and either a Gauss-Seidel or Jacobi relaxation in sequence. The Laplacian operator is a seven point, variable-coefficient stencil derived from a finite-volume calculation, while the Helmholtz and relaxation codes nominally scale and add vectors (grids) together. In the code, these become nested loops over the box list, and the spatial dimensions update either every or every other element.

\section{Optimizations for Smooth}

Starting with the baseline miniGMG code of Figure 3(a), we now describe how our compiler transforms the code to realize both vertical and horizontal communication-avoiding optimizations. The compiler first fuses the multiple smooth operators together. In the case of GSRB relaxation, the control flow guarding the update to phi may prevent fusion in some compilers. Incorporating data-flow analysis allows us to fuse the loops safely by contracting the iteration space of the first two statements (see next section). Fusion is itself a vertical communication-avoiding optimization, since the results computed by one operator will remain in cache when used as input by the next operator; an additional communication-avoiding optimization is to replace the array temp with a scalar and not write it back to memory on completion. The compiler generates the code in Figure 4(a), with placeholders for the statements corresponding to Figure 3(a). 


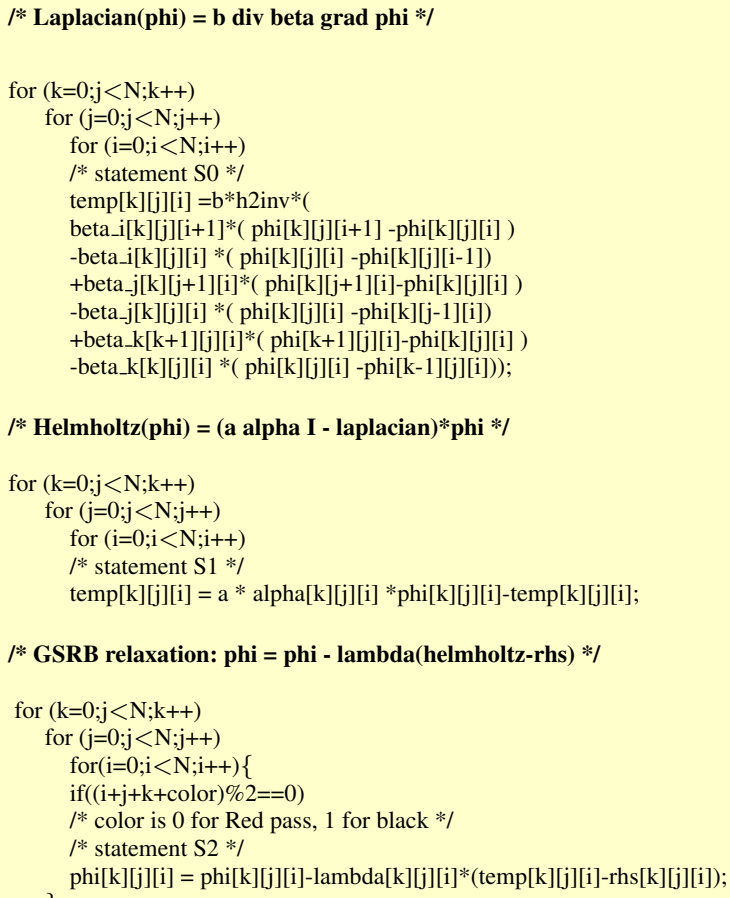

/* Helmholtz $($ phi $)=($ a alpha I - laplacian $) *$ phi */

for $(\mathrm{k}=0 ; \mathrm{j}<\mathrm{N} ; \mathrm{k}++)$

(a) Smooth operator with Gauss-Seidel Red-Black.

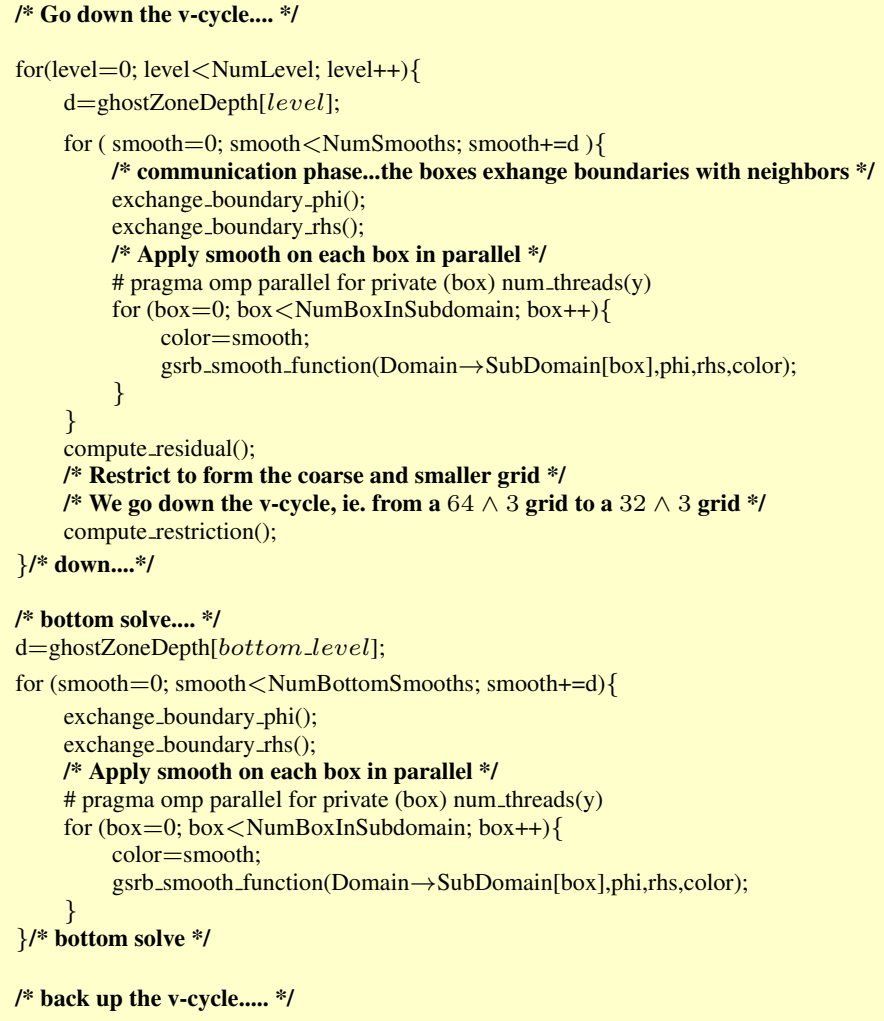

(b) Pseudo-code for a single iteration of the V-cycle.

Figure 3. Baseline smooth code using Gauss-Seidel Red-Black and outer V-cycle code that includes domain decomposition.

In the pseudo-code that invokes smooth in Figure 3(b), for each subdomain, the smooth operation is called on all the boxes in parallel. After the smooth operator, the boxes go through a communication phase, where they exchange boundary data with their neighbors. An important communication-avoiding optimization is to create ghost zones, which replicate some of the input data across processes and threads. Through the use of ghost zones, the computation can perform several sweeps of the grid per communication step, trading off increased computation for lower communication costs. For the seven-point second-order stencil we consider for our study, an $N$-deep ghost zone allows $N$ sweeps of the grid between communication. For higher order stencils, the ghost zone depth required increases with order.

We have added a domain-specific compiler transformation to automatically generate a loop over the depth of ghost zones, and modify the iteration space of the operator accordingly to perform the redundant computation. Each sweep of the grid consumes a layer of the ghost zone, and this gives rise to a hyper-trapezoidal iteration space of computation, where the volume shrinks in all dimensions on every smooth to perform computation only on valid data. The GSRB smooth has a red and a black pass, where the points updated depend on their coordinate and the value of color. As shown in Figure 3(b), for a grid with a one-deep ghost zone, the value of color is updated every time smooth is called. Adding ghost zones requires that the code track the value of color and modify the if-condition accordingly. The compiler merges the ifcondition into the loop bounds of the innermost loop to generate the transformed code in Figure 4(b).
Adding ghost zones increases DRAM traffic from multiple sweeps over the grid; this vertical communication can be reduced by a streaming strategy called wavefront [30]. A number of planes (up to the number of ghost zones) can be held in memory at once, as shown in Figure 2, and the number of sweeps of the grid reduced by the depth (the number of planes). Keeping multiple planes in memory increases the working set, which may exceed on-chip memory. We generate multi-threaded code via OpenMP to share planes across threads and reduce the working set per thread. Larger grids have a bigger working set than the smaller grids down the Vcycle, which suggests that the system should assign more threads per box for the larger grids, and fewer threads for the smaller grids - ultimately the thread distribution is optimized via autotuning. The threaded wavefront code is in Figure 4(c), which assigns $x$ OpenMP threads per box (intra-box parallelism). Outer-loop parallelism in the harness code of Figure 3(b) assigns $y$ threads to each box (inter-box parallelism).

The autotuning phase tunes the ghost zone depth at each level (ghostZoneDepth[level] in Figure 3(b)) and the number of threads $x$ and $y$ controlling intra- and inter-box parallelism, respectively. During the tuning process, constant values for these are bound during code generation, resulting in very efficient context-specific specialized code such as in Figure 4(d).

\section{Compiler Implementation}

We now describe the compiler implementation that generates the desired code from the previous section. This compiler is domain specific, in that it employs optimizations that are designed for 


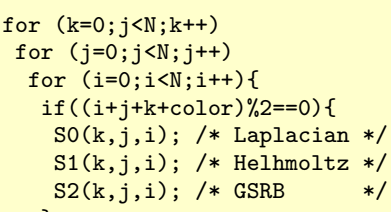

(a) Fused operators.

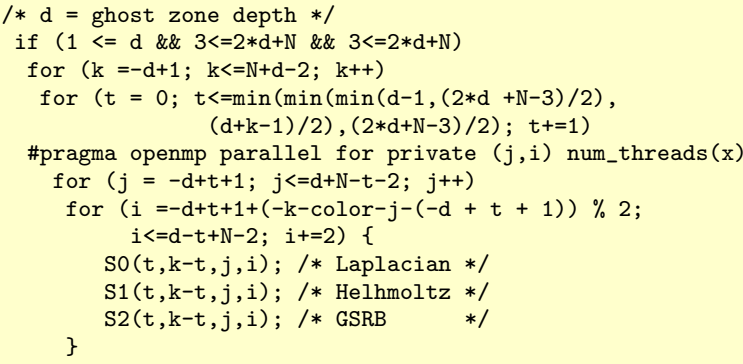

(c) Wavefront and threading.

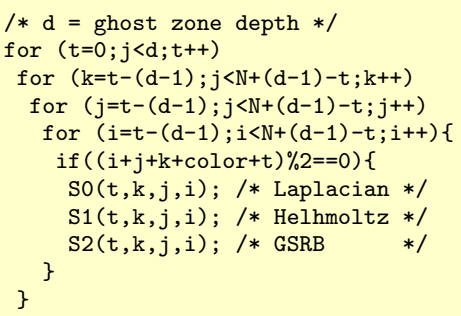

(b) After adding ghost zones.

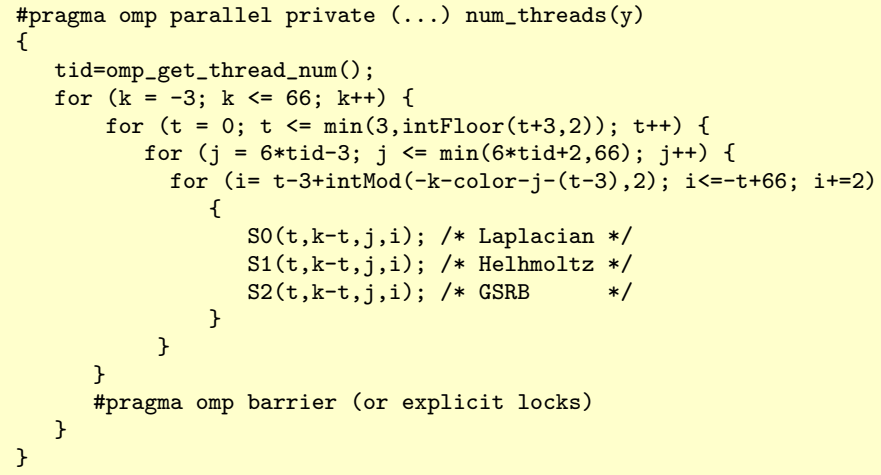

(d) Final code specialized for box size $64^{3}$, 4-deep ghost zone and 12 threads.

Figure 4. Steps of optimization process.

multigrid applications. The input to the compiler is the code excerpt shown in Figure 3. We performed the implementation using CHiLL, which is a polyhedral transformation and code generation framework [4]. The remainder of this section describes the abstractions used in CHiLL, how these abstractions are used to represent the transformations described in the previous section, the algorithm that performs these transformations, and how autotuning is employed to optimize the code for different architectures.

\subsection{Abstractions in CHiLL}

CHiLL is polyhedral transformation and code generation framework designed specifically for autotuning. A polyhedral model represents each statement's execution in the loop nest as a lattice point in the space constrained by loop bounds, known as the iteration space. Then a loop transformation can be simply viewed as mapping from one iteration space to another, and various transformations can be composed. CHiLL manipulates iteration spaces derived from the original program, using a data dependence graph as an abstraction to reason about the safety of the transformations under consideration [1].

In a polyhedral model, optimized code is generated from the rewritten iteration spaces by scanning the polyhedra representing the iteration spaces of an optimized loop nest from the first dimension to the last. The quality of the generated code directly impacts performance. Therefore, at the heart of CHiLL is a code generator called CodeGen+ that has advanced the state of the art in polyhedral scanning in the presence of conditionals [3], as arise in the GSRB code. CodeGen+ seamlessly combines iteration spaces and guards through a specialized polyhedral AST, as detailed elsewhere [3].

The input to CHiLL is a source code written in C or Fortran, and optionally, a transformation recipe describing the set of transformations to be composed to optimize the provided source [12].
This recipe can be written by an expert programmer, or derived automatically by a compiler decision algorithm [14].

\subsection{Communication-Avoiding Transformations}

As we describe each of the communication-avoiding transformations for GMG, we show how the previously-described abstractions are manipulated by CHiLL.

Operator fusion: The input code in Figure 3 consists of three statements S0, S1, and S2, that correspond to the three smooth operators Laplacian, Helmholtz and GSRB, respectively. Once parsed by CHiLL, the iteration spaces corresponding to these operators are as follows:

S0 : $=\{[\mathrm{k}, \mathrm{j}, \mathrm{i}]: 0 \leq \mathrm{k}<\mathrm{N} \& \& 0 \leq \mathrm{j}<\mathrm{N} \& \& 0 \leq \mathrm{i}<\mathrm{N}\}$;

$\mathrm{S} 1:=\{[\mathrm{k}, \mathrm{j}, \mathrm{i}]: 0 \leq \mathrm{k}<\mathrm{N} \& \& 0 \leq \mathrm{j}<\mathrm{N} \& \& 0 \leq \mathrm{i}<\mathrm{N}\}$;

$\mathrm{S} 2:=\{[\mathrm{k}, \mathrm{j}, \mathrm{i}]: 0 \leq \mathrm{k}<\mathrm{N} \& \& 0 \leq \mathrm{j}<\mathrm{N} \& \& 0 \leq \mathrm{i}<\mathrm{N} \& \&$ $\mathrm{k}+\mathrm{j}+\mathrm{i}+2 \alpha+$ color $=0\}$

Note that S2 has an additional term in its iteration space related to checking the color for the current element. Operator fusion falls out implicitly from the iteration space alignment algorithm, which attempts to carve out a unified iteration space for the imperfect loop nest of the original code [4]. With smooth operators such as Jacobi, iteration space alignment is performed by default in CHiLL. In GSRB, the difficult challenge is to rule out any fusion-preventing dependences when the iteration spaces are not a perfect match.

By default, CHiLL reports a fusion-preventing dependence between S2 and S0 related to the reads and writes of phi. However, we make the observation that the iteration spaces for the Laplacian and Helmholtz operators (statements S0 and S1) may compute values of temp that are never used by the GSRB of S2. Array data-flow analysis can be used to analyze the iteration spaces and access expressions and derive a conservative approximation of the elements 
of temp defined in S0 and S1 and used in S2 [9]. Our compiler determines that the array region read by $\mathrm{S} 2$ is a proper subset of the regions defined by S0 and S1. Since temp is a local variable redefined on every sweep and it is not live after the smooth operator is completed, it is safe to contract the iteration spaces of S0 and S1 to match that of S2. After the compiler contracts the iteration space, the fusion-preventing dependences are eliminated and CHiLL is able to safely fuse the loops. The iteration space contraction used here is an example of a domain-specific optimization that was proven safe by the compiler, but is more likely to be profitable for GMG operators where Red-Black conditional execution is common.

In the fused code, the compiler recognizes that array temp is a local variable, and does not need to be rewritten back to memory. Because there are no dependences on temp crossing iteration boundaries, scalar replacement is then employed to make this a scalar that is overwritten on each iteration of the loop.

Introducing ghost zones: Once fused, the iteration spaces from the previous section end up with a combined iteration space that matches that of statement S2. We observe that introducing ghost zones as in the previous section is really just introducing a new loop $t$ and changing the bounds for each of the loops in the fused loop nest to compute ghost regions and generate a hyper-trapezoidal iteration space.

Due to the presence of the if-condition in the GSRB smooth, the iteration space is a hyper-trapezoid with holes. The iteration space IS has two distinct components, arising from the loop nest and also the relation $(\mathrm{k}+\mathrm{j}+\mathrm{i}+2 \alpha+\mathrm{color}=0)$ which represents the ifcondition; the iteration space is the conjunction of these terms. We added a new domain-specific transformation add_ghost_zones, which maps the old iteration space with the new loop $t$ using the following mapping:

IS : iteration space of the input loop nest

IS $^{\prime}$ : iteration space in the modified loop nest

map $:=\left\{[\mathrm{k}, \mathrm{j}, \mathrm{i}] \rightarrow\left[\mathrm{t}, \mathrm{k}^{\prime}, \mathrm{j}^{\prime}, \mathrm{i}^{\prime}\right]:\right.$

$0 \leq \mathrm{t}<\mathrm{d} \& \& \mathrm{k}-\mathrm{d}+1+\mathrm{t} \leq \mathrm{k}^{\prime}<\mathrm{k}+\mathrm{d}-\mathrm{t} \& \&$

$\left.\mathrm{j}-\mathrm{d}+1+\mathrm{t} \leq \mathrm{j}^{\prime}<\mathrm{j}+\mathrm{d}-\mathrm{t} \& \& \mathrm{i}-\mathrm{d}+1+\mathrm{t} \leq \mathrm{i}^{\prime}<\mathrm{i}+\mathrm{d}-\mathrm{t}\right\}$

$\mathrm{IS}^{\prime}:=\operatorname{map}($ IS );

The variable color gets updated with every sweep of the grid, so its value will also be affected by the additional loop. For this purpose, we apply another mapping to color:

$\operatorname{map}^{\prime}:=\{$ [color $] \rightarrow[$ color $\left.+\mathrm{t}]\right\}$

This will cause the value of color to be updated everywhere it appears, including within the statements. Although in our current implementation this relation is provided to the implementation, it could be derived automatically through analysis or domain knowledge. This gives a new relation $(\mathrm{k}+\mathrm{j}+\mathrm{i}+2 \alpha+$ color $+\mathrm{t}=0)$ for the ifcondition. The conjunct of the new loop-nest iteration space and the new term gives us the final modified iteration space.

Wavefront and Multithreading: The compiler next generates a wavefront computation [30] using a loop skew and permute, skewing the outermost loop which sweeps the grid, loop k in Figure 4(b), against the smooth iteration loop added in the last optimization. Skewing is used to break a dependence that would otherwise prevent permute, using an integer factor in each dimension that depends on the dependence distance in the stencil operation. The $\mathrm{k}$ and $t$ loops are then permuted. The $j$ loop is then tiled and each tile is assigned to an OpenMP thread. We can use an OpenMP barrier or generate explicit locks to synchronize the threads. Code with explicit locks achieves higher performance.

Autotuning Opportunities: We employ autotuning for two sets of parameters:

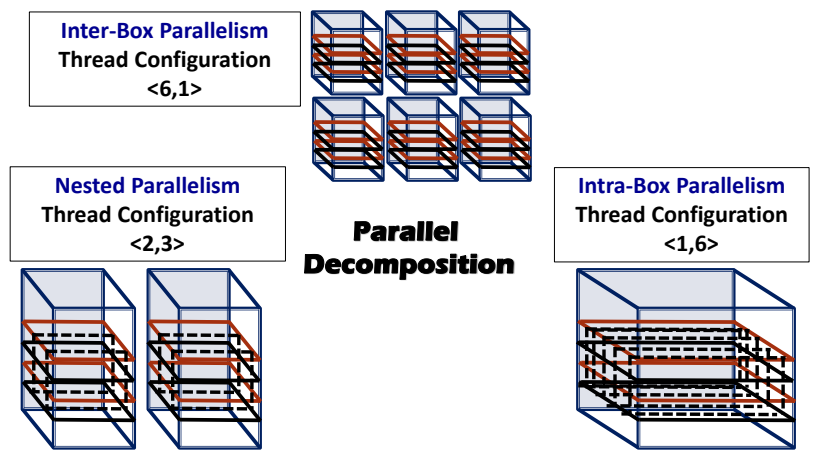

Figure 5. Figure shows possible thread decompostion on Hopper, which has 6-cores per chip. All the boxes in a subdomain may work in parallel, or only one maybe computed with all the theads working collaboratively on it, or nested parallelism can be used.

- Ghost zone and wavefront depth: The ghost zone depth governs both amount of redundant computation performed, and frequency of MPI communication. In the current code generation strategy, the ghost zone depth and number of planes in the wavefront are identical. As memory bandwidth is a key limitation only for larger box sizes, the optimal value for ghost zone depth varies for different box sizes in the V-cycle.

- Multithreading: As shown in Figure 5, the possible multithreading strategies are inter-box, intra-box and nested parallelism, where $x$ boxes are computed in parallel, and $y$ threads collaboratively work inside each box. The baseline implementation uses inter-box parallelism, where one thread works on a box. As box size varies across the V-cycle, the number of threads working collaboratively on a box also varies.

Putting it together: As the goal of this work is to develop domain-specific optimization techniques for GMG, the compiler algorithm can be specialized for MG implementations that involve composing a set of operators together. The code for the smooth operators was generated by instantiating a template transformation recipe that is then applied to the input code to generate the optimized code. The recipe that is generated for the smooth that includes GSRB is the following:

original()

add_ghosts ([SO],L1,d) \#ghost depth is d

skew $([\mathrm{S} 0], \mathrm{L} 2,[1,1]) \quad$ \#skew L2 : L2 <- 1.L1 + 1.L2

permute ([L2, L1, L3, L4]) \#new loop order

tile (SO, L3, TJ, 2, counted) \#tile loop L3 (j-loop)

gen_omp_parallel_region(locks,y)\#parallelize with y threads per box

The commands in this recipe refer to applying a transformation to a statement at a particular loop level. Once fused, the same transformations are applied to the set of statements S0, S1 and S2 when applied to S0. The tile command tiles the j-loop, and each tile is assingned to a thread. The command gen_omp_parallel_region generates parallel code. The boolean locks is used to generate OpenMP code with either an OpenMP barrier or low level explicit locks. During code generation, the value for ghost zone depth and box size and number of threads are bound to constants.

\section{Experimental Results}

In this section we present an overview of our experimental platforms and a detailed analysis of our performance results.

Through an external python script, autotuning varies $d$ from 1 to $2 * n$ where $\mathrm{n}$ ranges from 1 to 2 , and the thread values $x$ and $y$ ( $x * y=6$ for Hopper and 12 for Edison) for the fused and wavefront 


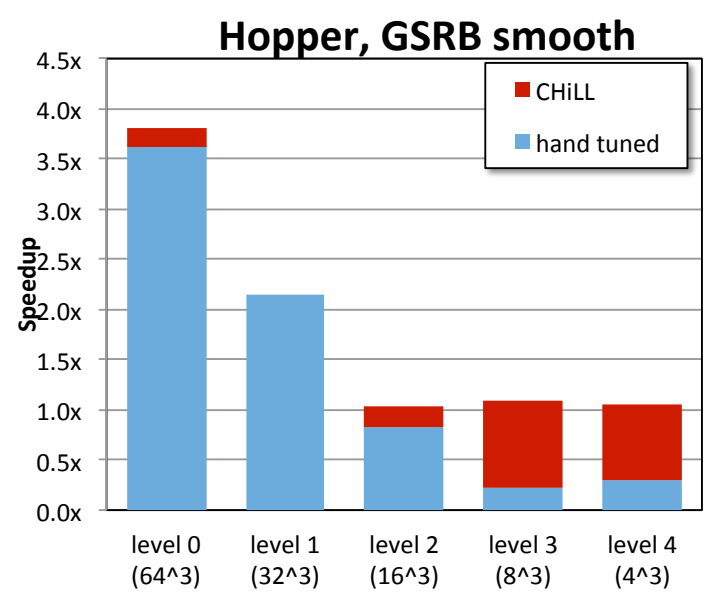

(a)

\begin{tabular}{|l|l|l|l|}
\hline Box Size & $\begin{array}{l}\text { Ghost Zone } \\
\text { Depth }\end{array}$ & $\begin{array}{l}\text { Thread } \\
\text { Decomposition }\end{array}$ & Code Variant \\
\hline $64^{\wedge 3}$ & 4 & $<2,3>$ & Wavefront \\
\hline $32^{\wedge 3}$ & 4 & $<2,3>$ & Wavefront \\
\hline $16^{\wedge 3}$ & 2 & $<6,1>$ & Wavefront \\
\hline $8^{\wedge 3}$ & 2 & $<6,1>$ & Fused \\
\hline $4^{\wedge 3}$ & 4 & $<6,1>$ & Fused \\
\hline
\end{tabular}

(c)

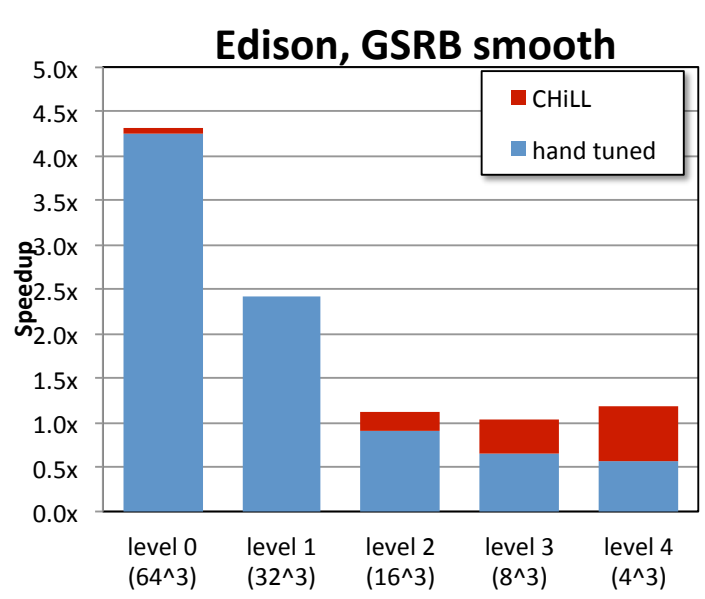

(b)

\begin{tabular}{|l|l|l|l|}
\hline Box Size & $\begin{array}{l}\text { Ghost Zone } \\
\text { Depth }\end{array}$ & $\begin{array}{l}\text { Thread } \\
\text { Decomposition }\end{array}$ & Code Variant \\
\hline $64 \wedge 3$ & 4 & $<4,3>$ & Wavefront \\
\hline $32^{\wedge 3}$ & 4 & $<4,3>$ & Wavefront \\
\hline $16 \wedge 3$ & 2 & $<12,1>$ & Wavefront \\
\hline $8 \wedge 3$ & 2 & $<12,1>$ & Fused \\
\hline $4 \wedge 3$ & 2 & $<12,1>$ & Fused \\
\hline
\end{tabular}

(d)

Figure 6. Speedups relative to the baseline code with tuned fusion/wavefront, ghost zone depth and threading as a function of level in the V-cycle for the $256^{3}$ problem for smooth time $(\mathrm{a}, \mathrm{b})$ and code configuration $(\mathrm{c}, \mathrm{d})$.

variants. Tile size TJ is related to the number of threads $y$ and loop bounds (box size and ghost zone depth). This search space can be explored in a few hours, but with additional optimizations, sophisticated search algorithms are needed to increase the efficiency of the search (e.g., [23]). The resulting application will select the best implementation among precompiled choices.

\subsection{Evaluated Platforms}

We evaluate the benefits of our compiler technology on two commodity processor architectures similar to those used by Williams et al. in [29]. Our code was compiled with icc version 13.0.1 with flags -O3 -fno-alias -fno-fnalias (Hopper:-msse3, Edison:xSSSE3).

Edison: is a Cray XC30 MPP at NERSC. Each node contains two 12-core Xeon Sandy Bridge chips. Each chip has four DDR3-1866 memory controllers.Each superscalar out-of-order core implements the 4-way AVX SIMD instruction set and includes both a $32 \mathrm{~KB} \mathrm{L1}$ and a $256 \mathrm{~B}$ L2 cache. The cores on a chip are connected by a ring to a 30MB L3 cache. The relatively large last-level cache makes capturing locality easier for $64^{3}$ boxes. Experiments were run on the pre-production Edison Phase II.

Hopper: is a Cray XE6 MPP at NERSC. Each node is in effect four 6-core Opteron chips each with two DDR3-1333 memory controllers. There are thus four (non-uniform memory access) NUMA nodes per compute nodes. Each superscalar out-of-order core implements the 2-way SSE3 SIMD instruction set and includes both a $64 \mathrm{~KB} \mathrm{L1}$ and a $512 \mathrm{~KB}$ L2 cache, while each socket includes a $6 \mathrm{MB}$ L3 cache with $1 \mathrm{MB}$ reserved for the probe filter. The relatively small last-level cache makes capturing locality difficult on fine grid operations.

\subsection{GSRB Demonstration and Analysis}

To provide a base case, we first ran the $256^{3}$ problem using a GSRB relaxation (from Figure 3 ) with one process per NUMA node on Edison and Hopper. Figure 6 presents tuned performance for the smooth operation (top) and the code configurations selected by the autotuner to achieve the best performance (bottom). Performance (speedup) has been normalized to the baseline implementation on either Hopper or Edison. Figure 7 shows the overall speedup for the MG solver on the finest box $\left(64^{3}\right)$.

By fusing the operators in smooth, the compiler yields dramatic speedups in smooth time on the finer grids. However, the benefit degrades as one descends through the V-cycle. This effect is caused by the fact that in the baseline implementation, the working set of each triply-nested loop within smooth exceeds cache capacity for the fine grids. As a result, to construct the Helmholtz, the Laplacian must be refetched from DRAM. Eventually this working set becomes small enough that it fits in the last-level cache at which point the disparity between on chip-bandwidth and compute capability limits the performance benefit.

Our compiler may now insert additional ghosts zones and generate a wavefront-based update for the smooth operation. Once again, on the finer (large) grids, the performance benefit arises from trading DRAM accesses for cache accesses. The benefit of a wavefront computation is likely smaller on Edison as its massive $20 \mathrm{MB}$ cache is nearly capable of capturing the locality required on the finest grids.

The local computation (smooth) for the wavefront performs poorly on the coarsest grids as the size of those grids has ballooned from approximately $12 \mathrm{~KB}$ per box to nearly $100 \mathrm{~KB}$. The increase in data movement impedes performance. Although smooth may be 


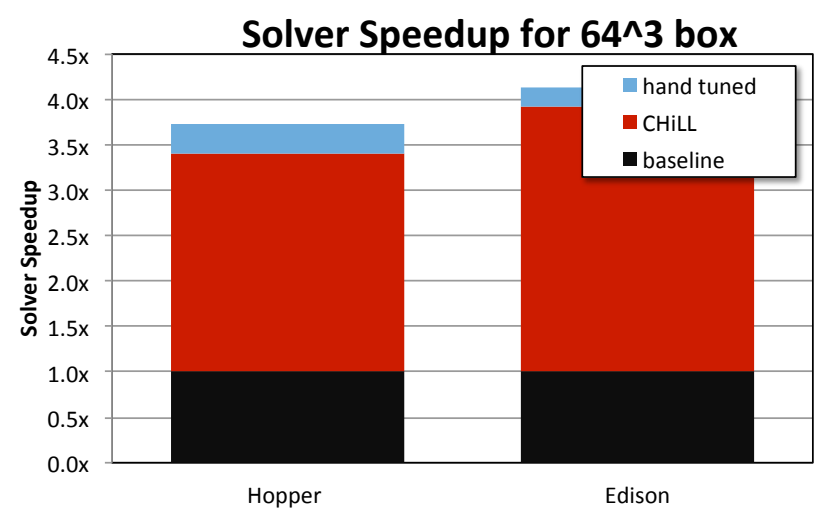

Figure 7. Overall speedup for the multigrid solver on the finest grid compared to baseline and hand-optimized code.

slower on the coarse grids, the reduction in inter-box communication can actually accelerate the multigrid operations. We see this benefit applies to all levels of the V-cycle. However, where as on the coarsest grids, the benefit of reduced inter-box communication compensates for reduced smooth performance, the additional cost of communication actually impedes smooth performance on the fine grids. This presents an interesting tuning space for our compiler infrastructure, which was addressed via autotuning.

Figure 6 presents smooth performance as a function of fusion, wavefront, ghost-zone depth and parallel decomposition. We see that for fine large grids $(64,32,16)$ it is profitable to use a wavefront computation, but for smaller grids fused code with smaller ghost zone depths are better. Results also show that for finer grids $(64,32)$ with larger working sets, it pays to have collaborative nested threading, but for smaller boxes overhead of collaborative threading impedes performance.

\subsection{Overall Speedup}

As the focus of this paper was the time-dominating smooth operation, we see the biggest gains there: speedups of $3.81 \times$ and $4.32 \times$ over the baseline, and within $1.05 \times$ and $1.01 \times$ of hand-tuned performance on Hopper and Edison, respectively. Figure 7 summarizes the overall speedup for the finest box $\left(64^{3}\right)$ CHiLL attains over the baseline optimized by the icc compiler, and compares with the hand-tuned code of Williams et al. [29]. As smooth is only one component in a multigrid solver, the benefits are amortized in the full GMG application.

\section{Related Work}

In the past, operations on large structured grids could easily be bound by capacity misses in cache, leading to a variety of studies on blocking and tiling optimizations $[6,8,15,19,20,27,28]$. However, a number of factors have made such approaches progressively obsolete on modern platforms. On-chip caches have grown by orders of magnitude and are increasingly able to capture sufficient locality for the fixed box sizes associated with typical MG methods. The rapid increase in on-chip parallelism has also quickly outstripped available DRAM bandwidth resulting in bandwidth-bound performance.

Thus, in recent years, numerous efforts have focused on increasing temporal locality by fusing multiple stencil sweeps through techniques like cache oblivious, time skewing, wavefront or overlapped tiling [7, 10, 11, 17, 18, 21, 25, 26, 31, 32, 34]. Many of these efforts examined 2D or constant-coefficient problems - features rarely seen in real-world applications.

Chan et al. explored how, using an autotuned approach, one could restructure the MG V-cycle to improve time-to-solution in the context of a 2D, constant-coefficient Laplacian [2]. This approach is orthogonal to our implemented optimizations and their technique could be incorporated in future work.

Closely related work from Treibig implements a 2D GSRB on SIMD architectures by separating and reordering the red and black elements [24], additionally a 3D multigrid on an IA-64 (Itanium) is implemented via temporal blocking.

The most closely related work are domain-specific compilers for parallel code generation from a stylized stencil specification [5, $22,33]$ or from a code excerpt [13]. Pochoir uses a cache oblivious strategy, which limits the control over the code generation [22]. The other compilers introduce parallelism and ghost regions through tiling and expanding both the data set and the tile size, rather than starting with already parallel code $[13,33]$. These tiling approaches do not produce the hyper-trapezoidal loop nests of this paper, but rather compute and then ignore some incorrect results. None of these approaches appear capable of supporting the optimization of a collection of operators, particularly if GSRB is included.

Our work expands on all of these efforts by providing a unique set of optimization strategies for multi- and manycore architectures.

\section{Conclusion}

In this paper, we have described autotuning compiler technology to automate communication-avoiding optimizations for the smooth operator in a geometric multigrid computation. Our results show that the optimizations lead to speedups as high as $4 \times$, and that different optimization strategies are needed at different levels of the V-cycle. As compared with related domain-specific compiler research, our work is distinguished by focusing on the needs of scalable GMG applications, starting with a parallel code and considering the composition of smooth operators including the complex Gauss-Seidel Red-Black operator. As compared with manually-tuned codes, the automatically-generated code captures the communication-avoiding optimizations, while attaining equivalent performance.

\section{Acknowledgments}

Authors from Lawrence Berkeley National Laboratory were supported by the DOE Office of Advanced Scientific Computing Research under contract number DE-AC02-05CH11231. Utah researchers were partially supported by DOE award DE-SC0008682 and National Science Foundation award CCF-1018881. This research used resources of the National Energy Research Scientific Computing Center, which is supported by the Office of Science of the U.S. Department of Energy under Contract No. DE-AC0205CH11231.

\section{References}

[1] R. Allen and K. Kennedy. Optimizing Compilers for Modern Architectures: A Dependence-based Approach. Morgan Kauffman, 2002.

[2] C. Chan, J. Ansel, Y. L. Wong, S. Amarasinghe, and A. Edelman. Autotuning multigrid with petabricks. In Proc. of the Conference on High Performance Computing Networking, Storage and Analysis, SC '09, Nov. 2009.

[3] C. Chen. Polyhedra scanning revisited. In Proc. of the 33rd ACM SIGPLAN conference on Programming Language Design and Implementation, PLDI '12, 2012.

[4] C. Chen, J. Chame, and M. Hall. CHiLL: A framework for composing high-level loop transformations. Technical Report 08-897, University of Southern California, June 2008. 
[5] M. Christen, O. Schenk, and H. Burkhart. Patus: A code generation and autotuning framework for parallel iterative stencil computations on modern microarchitectures. In Parallel Distributed Processing Symposium (IPDPS), 2011 IEEE International, pages 676-687, 2011.

[6] K. Datta, M. Murphy, V. Volkov, S. Williams, J. Carter, L. Oliker, D. Patterson, J. Shalf, and K. Yelick. Stencil computation optimization and auto-tuning on state-of-the-art multicore architectures. In Proc. 2008 ACM/IEEE Conf. on Supercomputing (SC 2008), 2008.

[7] K. Datta, S. Kamil, S. Williams, L. Oliker, J. Shalf, and K. Yelick. Optimization and performance modeling of stencil computations on modern microprocessors. SIAM Review, 51(1):129-159, 2009. . URL http://link.aip.org/link/?SIR/51/129/1.

[8] C. C. Douglas, J. Hu, M. Kowarschik, U. Rde, and C. Weiss. Cache optimization for structured and unstructured grid multigrid. Elect. Trans. Numer. Anal, 10:21-40, 2000

[9] P. Feautrier. Dataflow analysis of array and scalar references. International Journal of Parallel Programming, 20, 1991.

[10] M. Frigo and V. Strumpen. Evaluation of cache-based superscalar and cacheless vector architectures for scientific computations. In Proc. of the 19th ACM International Conference on Supercomputing (ICS05), Boston, MA, 2005.

[11] P. Ghysels, P. Kosiewicz, and W. Vanroose. Improving the arithmetic intensity of multigrid with the help of polynomial smoothers. Numerical Linear Algebra with Applications, 19(2):253-267, 2012. ISSN 1099-1506. . URL http://dx.doi .org/10.1002/nla.1808.

[12] M. W. Hall, J. Chame, C. Chen, J. Shin, G. Rudy, and M. M. Khan. Loop transformation recipes for code generation and auto-tuning. In Proc. of the 22nd International Workshop on Languages and Compilers for Parallel Computing, Oct 2009.

[13] J. Holewinski, L.-N. Pouchet, and P. Sadayappan. High-performance code generation for stencil computations on gpu architectures. In Proc. of the 26th ACM international conference on Supercomputing, ICS '12. ACM, 2012.

[14] M. Khan, P. Basu, G. Rudy, M. Hall, C. Chen, and J. Chame. A scriptbased autotuning compiler system to generate high-performance cuda code. ACM Trans. Archit. Code Optim., 9(4):31:1-31:25, Jan. 2013.

[15] M. Kowarschik and C. Wei. Dimepack - a cache-optimized multigrid library. In Proc. of the International Conference on Parallel and Distributed Processing Techniques and Applications (PDPTA 2001), volume I, pages 425-430. CSREA, CSREA Press, 2001.

[16] S. Krishnamoorthy, M. Baskaran, U. Bondhugula, J. Ramanujam, A. Rountev, and P. Sadayappan. Effective automatic parallelization of stencil computations. In Proc. of the 2007 ACM SIGPLAN conference on Programming language design and implementation, PLDI '07, New York, NY, USA, 2007. ACM.

[17] J. McCalpin and D. Wonnacott. Time skewing: A value-based approach to optimizing for memory locality. Technical Report DCS-TR379, Department of Computer Science, Rugers University, 1999.

[18] A. Nguyen, N. Satish, J. Chhugani, C. Kim, and P. Dubey. 3.5-D blocking optimization for stencil computations on modern CPUs and GPUs. In Proc. of the 2010 ACM/IEEE International Conference for High Performance Computing, Networking, Storage and Analysis, SC '10. IEEE Computer Society, 2010.

[19] G. Rivera and C. Tseng. Tiling optimizations for 3D scientific computations. In Proc. of SC'00, Dallas, TX, November 2000. Supercomputing 2000.

[20] S. Sellappa and S. Chatterjee. Cache-efficient multigrid algorithms. International Journal of High Performance Computing Applications, 18(1):115-133, 2004.

[21] Y. Song and Z. Li. New tiling techniques to improve cache temporal locality. In Proc. ACM SIGPLAN Conference on Programming Language Design and Implementation, Atlanta, GA, 1999.

[22] Y. Tang, R. A. Chowdhury, B. C. Kuszmaul, C.-K. Luk, and C. E. Leiserson. The pochoir stencil compiler. In Proc. of the 23rd ACM symposium on Parallelism in algorithms and architectures, SPAA '11, pages 117-128, New York, NY, USA, 2011. ACM. ISBN 978-1-
4503-0743-7. . URL http://doi .acm.org/10.1145/1989493. 1989508.

[23] A. Tiwari, C. Chen, J. Chame, M. Hall, and J. K. Hollingsworth. A scalable auto-tuning framework for compiler optimization. In International Parallel and Distributed Processing Symposium, Apr. 2009.

[24] J. Treibig. Efficiency improvements of iterative numerical algorithms on modern architectures. $\mathrm{PhD}$ thesis, University Erlangen, 2008.

[25] G. Wellein, G. Hager, T. Zeiser, M. Wittmann, and H. Fehske. Efficient temporal blocking for stencil computations by multicore-aware wavefront parallelization. In International Computer Software and Applications Conference, pages 579-586, 2009. .

[26] S. Williams, J. Shalf, L. Oliker, S. Kamil, P. Husbands, and K. Yelick. The potential of the Cell processor for scientific computing. In Proc. of the 3rd Conference on Computing Frontiers, 2006.

[27] S. Williams, J. Carter, L. Oliker, J. Shalf, and K. Yelick. Lattice Boltzmann simulation optimization on leading multicore platforms. In Interational Conference on Parallel and Distributed Computing Systems (IPDPS), Miami, Florida, 2008.

[28] S. Williams, L. Oliker, J. Carter, and J. Shalf. Extracting ultrascale lattice boltzmann performance via hierarchical and distributed auto-tuning. In Proc. of 2011 International Conference for High Performance Computing, Networking, Storage and Analysis, SC '11, 2011. ISBN 978-1-4503-0771-0.

[29] S. Williams, D. D. Kalamkar, A. Singh, A. M. Deshpande, B. Van Straalen, M. Smelyanskiy, A. Almgren, P. Dubey, J. Shalf, and L. Oliker. Optimization of geometric multigrid for emerging multiand manycore processors. In Proc. of the International Conference on High Performance Computing, Networking, Storage and Analysis, SC '12. IEEE Computer Society Press, 2012.

[30] M. Wolfe. Loop skewing: the wavefront method revisited. Int. J. Parallel Program., 15(4):279-293, Oct. 1986. ISSN 0885-7458. . URL http://dx.doi.org/10.1007/BF01407876.

[31] D. Wonnacott. Using time skewing to eliminate idle time due to memory bandwidth and network limitations. In Interational Conference on Parallel and Distributed Computing Systems, 2000.

[32] T. Zeiser, G. Wellein, A. Nitsure, K. Iglberger, U. Rude, and G. Hager. Introducing a parallel cache oblivious blocking approach for the lattice Boltzmann method. Progress in Computational Fluid Dynamics, 8, 2008. .

[33] Y. Zhang and F. Mueller. Auto-generation and auto-tuning of 3d stencil codes on gpu clusters. In Proc. of the Tenth International Symposium on Code Generation and Optimization, CGO '12, Mar. 2012.

[34] X. Zhou, J.-P. Giacalone, M. J. Garzarán, R. H. Kuhn, Y. Ni, and D. Padua. Hierarchical overlapped tiling. In Proc. of the Tenth International Symposium on Code Generation and Optimization, CGO '12, Mar. 2012. 